\title{
ON THE ENVELOPE OF A VECTOR FIELD
}

\author{
BERNARD MALGRANGE \\ UFR de Mathématiques, UMR 5582, Institut Fourier, Université Grenoble 1 - CNRS \\ 38402 Saint-Martin d'Hères Cedex, France \\ E-mail: bernard.malgrange@ujf-grenoble.fr
}

To Michael Singer

\begin{abstract}
Given a vector field $X$ on an algebraic variety $V$ over $\mathbb{C}$, I compare the following two objects: (i) the envelope of $X$, the smallest algebraic pseudogroup over $V$ whose Lie algebra contains $X$, and (ii) the Galois pseudogroup of the foliation defined by the vector field $X+d / d t$ (restricted to one fibre $t=$ constant). I show that either they are equal, or the second has codimension one in the first.
\end{abstract}

1. Introduction. This note is a simple exercise in the "non-linear differential Galois theory". I refer for this theory to [Ma2], or [Ma1] (but this last paper is written in an analytic context, and one should make the translation analytic $\rightarrow$ algebraic to recover the situation of [Ma2]).

Let $X$ be a complex algebraic variety (= a reduced scheme of finite type over $\mathbb{C}$ ), which I will suppose irreducible. As usual in this subject, I work birationnally, i.e. I can replace freely $X$ by an open dense Zariski subvariety. Therefore, I can suppose $X$ affine, non-singular, and even a finite étale covering $X \stackrel{p}{\rightarrow} U$ of $U=\mathbb{C}^{n}-Z, Z$ a closed hypersurface. In that situation, I denote by $x$ a (closed) point of $X$, and by $\left(x_{1}, \ldots, x_{n}\right)$ the coordinates of $p(x) \in U$. I call these data "étale coordinates" on $X$.

Let $\xi$ be a vector field on $X$; in étale coordinates, one has $\xi=\sum a_{i} \frac{\partial}{\partial x_{i}}, a_{i} \in \mathbb{C}[X]$ (= $a_{i}$ regular over $\left.X\right)$. Recall the following definition (see loc. cit.).

Definition 1.1. The envelope $E(\xi)$ of $\xi$ is the smallest (algebraic) pseudogroup on $X$ whose Lie algebra Lie $E(\xi)$ contains $\xi$ as solution.

Now, to $\xi$ is associated naturally a differential equation, which, in étale coordinates, is written $\frac{d x_{i}}{d t}=a_{i}(x)$. Instead of this equation, it is equivalent to consider, on $X \times \mathbb{C}$, the foliation $\left\{\omega_{i}=d x_{i}-a_{i} d t\right\}$ (the Frobenius condition is obviously satisfied here). This

2010 Mathematics Subject Classification: Primary 37C10; Secondary 34Lxx.

Key words and phrases: vector fields, differential Galois theory.

The paper is in final form and no version of it will be published elsewhere. 
foliation is also defined by the vector field $\xi+\frac{\partial}{\partial t}$, or any of its multiples. To this foliation is associated its the Galois pseudogroup, which is the smallest pseudogroup on $X \times \mathbb{C}$ whose Lie algebra contains as solutions the vectors tangent to the leaves, i.e. the multiples of $\xi+\frac{\partial}{\partial t}$. Take a point $a \in \mathbb{C}$; by restriction to $X \times\{a\}$, we obtain a pseudogroup on $X$, independent of $a$ (because $\xi+\frac{\partial}{\partial t}$ is fixed by translations in $t$ ). I will call this restriction, by abuse of terminology, the "Galois pseudogroup of $\xi$ ". I will denote it by $G(\xi)$, and its $D$-Lie algebra will be denoted by Lie $G(\xi)$.

A natural question is the following: what is the relation between $E(\xi)$ and $G(\xi)$ ? $A$ priori, they should not be very different. Before giving the general result, I will give a few very simple examples.

\section{Examples}

(i) $X=\mathbb{C}, \xi=\frac{\partial}{\partial x}$. Of course, $E(\xi)$ is the group of translations $G_{a}$ over $\mathbb{C}$, more precisely the pseudogroup whose solutions are the translations, i.e. the pseudogroup $x \mapsto \bar{x}, \frac{d \bar{x}}{d x}=1$. On the other hand, to determine $G(\xi)$, we must look at the foliation $\{d x-d t\}$ of $\mathbb{C}^{2}$. This foliation admits the first integral $x-t$. Therefore, the Galois pseudogroup of this foliation is given by $\bar{x}-\bar{t}=x-t$. Setting $\bar{t}=t=a$, we find that $G(\xi)$ reduces to the identity.

(ii) $X=\mathbb{C}$ (or $\left.\mathbb{C}^{*}\right), \xi=x \frac{\partial}{\partial x}$. The envelope is the pseudogroup associated to $G_{m}$, with equation (on $x \bar{x} \neq 0$ ) $x \frac{d \bar{x}}{d x}=\bar{x}$, or $\frac{d \bar{x}}{\bar{x}}=\frac{d x}{x}$. On the other hand, the foliation is given by $d x=x d t$, or better by the closed form $\frac{d x}{x}-d t$. The corresponding pseudogroup is given by $\frac{d \bar{x}}{\bar{x}}-d \bar{t}=\frac{d x}{x}-d t$. Setting $t=\bar{t}=a$, we find $\frac{d \bar{x}}{\bar{x}}=\frac{d x}{x}$; in other words we have $E(\xi)=G(\xi)$.

(iii) Take more generally $X=\mathbb{C}^{n}$, and take for $\xi$ a linear vector field $\xi=\sum a_{i j} x_{j} \frac{\partial}{\partial x_{i}}$, $a_{i j} \in \mathbb{C}$. I write $A=\left(a_{i j}\right)$, and I identify $\xi$ and $A$. To state the result, I need a few conventions. If $G$ is an algebraic subgroup of $G \ell(n)$ over $\mathbb{C}$, I will identify $G$ with the pseudogroup $\widetilde{G}$ on $\mathbb{C}^{n}$ whose solutions are the transformations of $G(\mathbb{C})$ (cf. [Ma1]). Similarly, I identify the Lie algebra Lie $G$ with the $D$-Lie algebra Lie $\widetilde{G}$.

Note that all the (closed) subpseudogroups of $\widetilde{G \ell(n)}$ are of the form $\widetilde{G}$ for a suitable $G$ (this result, easy, can be left to the reader).

Now, let $A=S+N,[S, N]=0$, be the standard decomposition of $A$ into a semisimple and nilpotent part. The result is the following

Proposition 2.1. If $\xi$ is semisimple, then $E(\xi)=G(\xi)$. If $\xi=S+N, N \neq 0$, then $G(\xi)=E(S)$. One has $E(\xi) \supset G(\xi)$, and Lie $E(\xi)=$ Lie $G(\xi)+\mathbb{C} \xi$ (or $\mathbb{C} N$, it is equivalent). In particular, $G(\xi)$ has codimension one in $E(\xi)$.

The proof can be left to the reader (work directly, or use the general results of the next sections). Just a few comments.

a) With the identification made above, $E(\xi)$ is simply the smallest algebraic subgroup of $G \ell(n)$ over $\mathbb{C}$ whose Lie algebra contains $\xi=A$. Its determination is essentially classical: use the Jordan normal form. The crucial point is given by the linear relations over $\mathbb{Q}$ of the eigenvalues of $A$. 
b) $G(\xi)$ is the Galois group of the system $\frac{d x_{i}}{d t}=\sum a_{i j} x_{j}$, in the sense of the usual linear theory [Ko], vP-Si] (cf. loc. cit.). Therefore, the determination is also classical (again use the Jordan normal form).

(iv) $X=\mathbb{C}^{2}, \xi=x y \frac{\partial}{\partial x}$.

First method, elementary. To find $E(\xi)$, one writes the flow of $\xi$, i.e. the solutions of $\frac{d \bar{x}}{d t}=\bar{x} \bar{y}, \frac{d \bar{y}}{d t}=0$ with the initial conditions $(x, y, t=0)$. One has $\bar{x}=x e^{y t}, \bar{y}=y$.

One fixes $t=a \in \mathbb{C}$, and one looks at the differential equations of $\bar{x}$ and $\bar{y}$ in terms of $x, y$, independently of $a$.

One has $\frac{\partial \bar{x}}{\partial x}=\frac{\bar{x}}{x}, \frac{\partial \bar{x}}{\partial y}=a \bar{x}$; to have an equation independent of $a$, one replaces the second equation by $d\left[\frac{1}{\bar{x}} \frac{\partial \bar{x}}{\partial y}\right]=0$.

To obtain the corresponding infinitesimal equations, one writes $\bar{x}=x+\varepsilon u, \bar{y}=y+\varepsilon v$, $\varepsilon^{2}=0$. One finds $v=0, x \frac{\partial u}{\partial x}=u, d\left[\frac{1}{x} \frac{\partial u}{\partial y}\right]=0$. The solutions are $C_{1} x \frac{\partial}{\partial x}+C_{2} x y \frac{\partial}{\partial x}$.

To find $G(\xi)$, we must write the flow in a slightly different way, i.e. write $\bar{x}, \bar{y}$ at time $\bar{t}$ with initial conditions $x, y$ at time $t$. This gives $\bar{x}=x e^{y(\bar{t}-t)}, \bar{y}=y$, therefore $\frac{\partial \bar{x}}{\partial x}=\frac{\bar{x}}{x}$, $\frac{\partial \bar{x}}{\partial y}=(\bar{t}-t) \bar{x}$. By restriction to $\bar{t}=t=a$, this gives $\frac{\bar{x}}{\partial x}=\frac{\bar{x}}{x}, \frac{\partial \bar{x}}{\partial y}=0$, then $\bar{x}=c \bar{x}, \bar{y}=y$, $c \in \mathbb{C}$. The solutions of Lie $G(\xi)$ are $c x \frac{\partial}{\partial x}, c \in \mathbb{C}$.

Second method. The preceding method has two inconveniences. First, it is not obvious $a$ priori that the equation obtained really defines pseudogroups (the verification, here easy, is only made a posteriori). Second, the method is very particular to equations which can be integrated explicitly, and does not generalize much.

I will give another method, which is similar to the one used in [Ma2], Chap. IV. The vector field $\xi$ is the Hamiltonian field of $h=y$ for the symplectic form $\sigma=\frac{1}{x y} d x \wedge d y$. Therefore the calculation of $G(\xi)$ is a special case of loc. cit., §IV.5. I just give the result.

The foliation is given by $\{d y, d x-x y d t\}$, with the first integral $y$. If we replace $d x-x y d t$ by $\omega=\frac{d x}{x}-y d t-t d y$, we get $d \omega=0$. Therefore, the pseudogroup (in $x, y, t)$ is obtained by fixing $y$ and $\omega$. By restriction to $t=a$, we obtain that $G(\xi)$ is defined by fixing $y$ and $\frac{d x}{x}$; this is equivalent to the result obtained by the first method.

To find $E(\xi)$ is a little more difficult. We will see later the following result: take $\varphi \in \mathbb{C}(t), \varphi \neq 0$, and denote by $G(\xi, \varphi)$ the restriction to $t=a$ (for a generic $a$ ) of the Galois pseudogroup of the foliation of $X \times \mathbb{C}$ defined by $\xi+\frac{1}{\varphi} \frac{\partial}{\partial t}$. Then $G(\xi, \varphi) \subset E(\xi)$, with equality for $\varphi$ "sufficiently general" (see $\$ 6$, Prop. 6.2).

The foliation is defined by $\{d y, \omega\}, \omega=\frac{d x}{x}-y \varphi d t$. One works as in loc. cit.

One has $d \omega=d y \wedge \omega_{1}, \omega_{1}=-\varphi d t$, and $d \omega_{1}=0$. One lifts these equations in a suitable frame bundle, by defining $\widetilde{\omega}=\omega+u d y, \widetilde{\omega}_{1}=\omega_{1}-d u, u \in \mathbb{C}$. One has again $d \widetilde{\omega}=d y \wedge \widetilde{\omega}_{1}, d \widetilde{\omega}_{1}=0$. Now, $\left(d y, \widetilde{\omega}, \widetilde{\omega}_{1}\right)$ give a prolongation of the foliation to $X \times \mathbb{C}_{t} \times \mathbb{C}_{u}$, and an "admissible pseudogroup" is obtained if one fixes $y, \widetilde{\omega}, \widetilde{\omega}_{1}$. It will be the Galois pseudogroup of the prolongation if the calculation is "minimal", i.e. if the class of $\omega_{1}$ in the relative de Rham cohomology of $X \times \mathbb{C}_{t} / \mathbb{C}_{y}$ is not zero. This will be the case if $\varphi d t$ is not exact i.e. $\varphi d t \neq d \psi, \psi \in \mathbb{C}(t)$. Suppose that this is the case: then, fixing $t=a$, one finds that the prolongation of $G(\xi, \varphi)$ to $X \times \mathbb{C}_{u}$ is obtained by fixing $y, \frac{d x}{x}+u d y, d u$. 
I leave to the reader to go down to $X$, and to verify that the result is the same as the one given above.

REMARK. The same method permits, more generally, by a suitable modification of [Ma2], $\S I V .5$, to calculate the envelope of a symplectic integrable vector field. I leave this to people who are interested.

3. First integrals. As in $\S 1$, let $X$ be a complex algebraic variety. I denote by $\mathbb{C}[X]$ (resp. $\mathbb{C}(X)$ ) its regular (resp. rational) functions. Let $\xi$ be a vector field on $X$. I denote by $K$ the field of first integrals of $\xi$, i.e. the subfield of $\mathbb{C}(X)$ annihilated by $\xi$. Similarly, I consider the vector field $\xi+\frac{\partial}{\partial t}$ on $X \times \mathbb{C}$, and I denote by $L \subset \mathbb{C}(X \times \mathbb{C})$ its field of first integrals. Obviously, $K \subset L$. One has the following result.

Proposition 3.1. The following statements are equivalent:

(i) One has $L \neq K$.

(ii) There exists $f \in \mathbb{C}(X)$ with $\xi f=1$. Furthermore $L=K(t-f)$.

The proof was suggested to me by a remark of J. A. Weil.

(ii) $\Rightarrow(i)$ is trivial. Now, let us suppose that $\xi+\frac{\partial}{\partial t}$ admits a first integral $g \in \mathbb{C}(X \times \mathbb{C})$ depending effectively on $t$; one can write $g=\frac{P}{Q}, P, Q \in \mathbb{C}(X)[t]$, relatively prime (as polynomials in $t)$. One has $\left(\xi+\frac{\partial}{\partial t}\right) P / P=\left(\xi+\frac{\partial}{\partial t}\right) Q / Q=c$, with $c \in \mathbb{C}(X)[t]$. Looking at the degrees in $t$, one shows that, actually, $c \in \mathbb{C}(X)$.

Let $P=a_{0}+a_{1} t+\cdots+a_{n} t^{n}, a_{n} \neq 0$. From $\left(\xi+\frac{\partial}{\partial t}\right) P=c P$, one deduces $\xi a_{n}=c a_{n}$; therefore $\left(\xi+\frac{\partial}{\partial t}\right)\left(\frac{P}{a_{n}}\right)=0$. Therefore $\bar{P}=\frac{P}{a_{n}}$ is a first integral of $\xi$. The same result holds for $Q$.

Now, note that $\frac{\partial}{\partial t}$ commutes with $\xi+\frac{\partial}{\partial t}$; therefore the $\left(\frac{\partial}{\partial t}\right)^{k} \bar{P}$ are also first integrals. Taking $k=n-1$, we get a first integral of the form $t-f$.

Finally, we must prove that $L=K(t-f)$. The preceding results show that it is sufficient to consider the first integrals which are polynomial in $t$. If $R=a_{0}+\cdots+a_{n} t^{n}$, $a_{n} \neq 0$, is such a first integral, $a_{n}$ is a first integral by the preceding calculation. Now, replace $R$ by $R-a_{n}(t-f)^{n}$ and proceed by recurrence.

EXAMPLE 3.2. Take the vector field $\xi=(x+y) \frac{\partial}{\partial x}+y \frac{\partial}{\partial y}$, corresponding to the Jordan matrix $\left(\begin{array}{ll}1 & 1 \\ 0 & 1\end{array}\right)$. One has $\xi\left(\frac{x}{y}\right)=1$. A similar result holds for $\xi=S+N$, with $N \neq 0$ (notations of $\$ 2$, iii)). On the other hand, I leave it to the reader to prove that $\xi f=1$ has no solution if $\xi=S$.

REMARK 3.3. If, instead of $\xi+\frac{\partial}{\partial t}$, we take $\xi+c t \frac{\partial}{\partial t}, c \in \mathbb{C}$, the same method gives the following result. Denote again by $K$ (resp. $L$ ) the field of first integrals of $\xi$ (resp. $\left.\xi+c t \frac{\partial}{\partial t}\right)$. Then, the following statements are equivalent

(i) $L \neq K$.

(ii) Let $\Lambda=\{k \in \mathbb{Z} ; \exists a \in \mathbb{C}(X)$, with $\xi a+c k a=0\}$. $\Lambda$ is obviously a subgroup of $\mathbb{Z}$. Then $\Lambda \neq\{0\}$. In that case, let $\ell>0$ be the generator of $\Lambda$, and let $a \in \mathbb{C}(X)$ satisfy $\xi a+c \ell a=0$. Then $a t^{\ell} \in L$, and $L=K\left(a t^{\ell}\right)$. 


\section{Prolongation}

(i) Let, as before, $E(\xi)$ be the envelope of $\xi$. I recall a method to describe it, given in Ca2. For $k \geq 0$, let $R_{k}(X)$, or $R_{k}$, be the space of $k$-frames on $X$, i.e. of invertible $k$-jets $\left(\mathbb{C}^{n}, 0\right) \rightarrow X(n=\operatorname{dim} X)$. It is a principal bundle (or "torsor") on the group $\Gamma_{k}$ of invertible $k$-jets $\left(\mathbb{C}^{n}, 0\right) \rightarrow\left(\mathbb{C}^{n}, 0\right)$. Let $X \stackrel{p}{\rightarrow} U, U \subset \mathbb{C}^{n}$, and $\left(x ; x_{1}, \ldots, x_{n}\right)$ étale coordinates on $X$ as in $\$ 1$. Then the coordinates on $R_{k}(U)$ are $\left(x_{i, \alpha}\right), 1 \leq i \leq n,|\alpha| \leq k$, $|\alpha|=\alpha_{1}+\cdots+\alpha_{n}$, with $x_{i, 0}=x_{i}, \operatorname{det}\left(x_{i, j}\right) \neq 0$ and $R_{k}(X)=X \times_{U} R_{k}(U)$. On $\lim _{\leftarrow} R_{k}(U)$, put

$$
D_{i}=\sum_{j, d} x_{j, d+\varepsilon_{i}} \frac{\partial}{\partial x_{j, \alpha}} \quad \text { and } \quad D^{\alpha}=D_{1}^{\alpha_{1}} \cdots D_{n}^{\alpha_{n}}
$$

denote by the same letters the lifting of these operators to $R_{k}(X)$. Now, the vector field $\xi=\sum a_{i} \frac{\partial}{\partial x_{i}}$ on $X$ has a canonical lifting $R_{k} \xi$ on $R_{k}(X)$. This lifting is fixed by $\Gamma_{k}$; and, in étale coordinates,

$$
R_{k} \xi=\xi+\sum_{1 \leq|\alpha| \leq k} D^{\alpha} a_{i} \frac{\partial}{\partial x_{i, \alpha}}
$$

(see e.g. [O] $)$. Then the pseudogroup $E(\xi)$ on $X$ is defined by a collection of closed subvarieties $Z_{k} \subset J_{k}^{*}(X), J_{k}^{*}(X)$ the space of invertible jets of order $k$ from $X$ to $X$. (Maybe after restricting $X) Z_{k}$ is a subgroupoid of $J_{k}^{*}(X)$. This is equivalent to giving a $\widetilde{Z}_{k}$, equivalence relation in $R_{k}$ stable by $\Gamma_{k}$ (see [Ma1] or [Ma2]). After restricting again $X$, such an equivalence relation is given by a quotient $R_{k} \stackrel{\pi}{\rightarrow} S_{k}$, and one has $\widetilde{Z}_{k}=R_{k} \times_{S_{k}} R_{k}$ (see references in loc. cit.).

But, practically by definition, $\widetilde{Z}_{k}$ is the smallest equivalence relation on $R_{k}$ for which $R_{k} \xi$ is vertical, i.e. tangent to the fibers of $\pi$. In terms of first integrals, this means the following: if $K_{k}$ is the subfield of $\mathbb{C}\left(R_{k}\right)$ of first integrals, then $K_{k}$ is the field of functions $\mathbb{C}\left(S_{k}\right)$. Then, $E(\xi)=\left\{Z_{k}\right\}$ can be described by the successive first integrals of the $R_{k} \xi$ (observe that a first integral of $R_{k} \xi$ is also one for $R_{\ell} \xi, \ell \geq k$ ).

(ii) We will now give a similar description of $G(\xi)$. A priori, we could do the same thing with $X$ replaced by $X \times \mathbb{C}$, and $\xi$ replaced by the family of all the multiples of $\xi+\frac{\partial}{\partial t}$. But, as explained in Ma2 (see I.6 to I.8), it is sufficient to work with the transverse frame bundles of the foliation. Choosing $X \times \mathbb{C} \rightarrow \mathbb{C}$ as a transverse projection, this transverse frame bundle is identified with $R_{k} \times \mathbb{C}$.

The prolongation of the foliation to $R_{k} \times \mathbb{C}$ is given by the vector field $R_{k} \xi+\frac{\partial}{\partial t}$ : to prove that, it is sufficient to prove that the corresponding differential equations $\frac{d x_{i, \alpha}}{d t}=$ $D^{\alpha} a_{i}$ coincide with the variational equation of order $k$ (cf. loc. cit. I.8), which is obvious.

Let now $Z_{k}^{\prime} \subset J_{k}^{*}(X \times \mathbb{C})$ be the equations of order $k$ of the Galois pseudogroups of the foliation $\left\{\xi+\frac{\partial}{\partial t}\right\}$. We have a description of $Z_{k}^{\prime}$ similar to that one of $Z_{k}$. Call $I_{k}$ the subfield of $\mathbb{C}\left(R_{k} \times \mathbb{C}\right)$ of first integrals of $R_{k}+\frac{\partial}{\partial t}$. Choosing a general $a \in \mathbb{C}$, this field can be identified with its restriction to $\mathbb{C}\left(R_{k}\right)=\mathbb{C}\left(R_{k} \times\{a\}\right)$; and $Z_{k}^{\prime}$ is described by $L_{k}$ as $Z_{k}$ is by $K_{k}$.

(iii) Now, we are in a position to apply the results of $\$ 3$, by just replacing $\xi$ by $R_{k} \xi$ and $X$ by $R_{k}$. We obtain the following result: 
ThEOREM 4.1. (i) We have always $G(\xi) \subset E(\xi)$.

(ii) If $G(\xi) \not \subset E(\xi)$, there exists a $k \geq 0$ such that $L_{k} \neq K_{k}$. In that case, there exists an $f \in \mathbb{C}\left(R_{k}\right)$ satisfying $R_{k}(\xi) f=1$, and one has $L_{k}=K_{k}(f)$. If this is true for $k$, it is also true for $\ell \geq k$, with the same function $f$.

(I wrote $K_{k}(f)$ instead of $K_{k}(t-f)$; this is equivalent, $f$ being transcendental over $K$; otherwise, it would be a first integral by a classical lemma; see e.g. [Ro]).

ExAmples. In the linear case (Example 2(iii)) the dichotomy occurs already for $k=0$. But this is not always the case. For instance, if $\xi=x y \frac{\partial}{\partial x}$ the equation $\xi f=0$ has no solution, but the equation $\left(R_{1} \xi\right) f=1$ has one.

Explicitly, denoting by $\left(x, y ; x_{1}, y_{1}, x_{2}, y_{2}\right)$ the coordinates on $R_{1}(X)$, with here $X=\mathbb{C}^{2}$, we have

$$
R_{1} \xi=x y \frac{\partial}{\partial x}+\left(x_{1} y+x y_{1}\right) \frac{\partial}{\partial x_{1}}+\left(x_{2} y+x y_{2}\right) \frac{\partial}{\partial x_{2}}, \text { and } f=\frac{x_{1}}{x y_{1}} .
$$

This reflects the fact that (by both methods) we had to make a prolongation to order one to calculate $G(\xi)$.

REMARK 4.2. With $\xi+c t \frac{\partial}{\partial t}$ instead of $\xi=\frac{\partial}{\partial t}$, starting from Remark 3.3 and arguing as in $\$ 4$ we get the following result (with obvious notations, similar to those of Theorem 4.1.

(i) One has $G\left(\xi, c t \frac{\partial}{\partial t}\right) \subset E(\xi)$.

(ii) If $G\left(\xi, c t \frac{\partial}{\partial t}\right) \neq E(\xi)$, there exists a $k \geq 0$ such that $L_{k} \neq K_{k}, L_{k}$ the field of first integrals of $\xi+c t \frac{\partial}{\partial t}$. Then $0 \neq \Lambda=\left\{k \in \mathbb{Z} \mid \exists a \in \mathbb{C}\left(R_{k}\right), R_{k} \xi a+c k a=0\right\}$; let $\ell>0$ be the generator of $\Lambda$, and let $a \in \mathbb{C}\left(R_{k}\right)$ satisfy $R_{\ell} \xi a+c \ell a=0$. Then $L_{k}=K_{k}\left(a t^{\ell}\right)\left[=K_{k}(a)\right]$, and the same is true for all $m \geq k$.

5. Lie algebras. Consider again $\xi+\frac{\partial}{\partial t}$. It remains to analyze the relations between Lie $G(\xi)$ and Lie $E(\xi)$ when $G(\xi) \neq E(\xi)$.

For that purpose, we have to recall briefly the relation between Lie pseudogroups and their Lie algebras (cf. [Ma1] or [Ma2]). Let $X$ be a smooth $\mathbb{C}$-variety, $T=T_{X}$ its tangent bundle, and $\mathcal{O}_{X}$ (resp. $\Omega_{X}$ ) the sheaf of regular functions (resp. 1-forms) on $X$. The ingredients are as follows.

(i) Denote by $J_{k} T$ the space of $k$-jets of sections of $T$, and by $J_{k}^{*}(X)$ the groupoid of $k$-jets of invertible maps from $X$ to $X$. Then $J_{k} T$ is canonically isomorphic to the normal bundle along the identity of $J_{k}^{*}(X)$.

(ii) Denote by $D_{k}$ the sheaf of linear differential operators of order $\leq k$ on $X$, and put $D=\bigcup D_{k}$. Then $D_{k}$ is an $\mathcal{O}_{X}$-bimodule, and $J_{k}(T)$ is the vector bundle associated to $D_{k} \otimes \mathcal{O}_{X} \Omega_{X}$ by the contravariant correspondence "vector bundles" $\leftrightarrow$ "coherent sheaves" (cf. e.g. [Gr]). In particular, the sheaf $\underline{J_{k} T}$ of sections of $J_{k} T$ is the dual over $\mathcal{O}_{X}$ of $D_{k} \otimes \mathcal{O}_{X} \Omega_{X}$.

(iii) Let $R_{k} \stackrel{\pi}{\rightarrow} X$ the frame bundle of $X$ of order $k$ and $T\left(R_{k}\right)$ its tangent bundle. Then the sections of $J_{k} T$ are canonically isomorphic to the sections of $T\left(R_{k}\right)$ stable by $\Gamma_{k}$ (definition in 4 . Localizing over $X$, one gets an isomorphism of sheaves $\underline{J_{k} T} \sim\left[\pi_{*} T\left(R_{k}\right)\right]^{\Gamma_{k}}$. Of course, one has also a similar result for the fibers over a point $a \in \bar{X}$. 
Denoting by $\rho$ the map $\underline{J_{k} T} \rightarrow \pi_{*} T\left(R_{k}\right)$, one has, in particular, $\rho\left(j_{k} \xi\right)=R_{k} \xi$ for a vector field $\xi$ on $X$. I leave to the reader to give the explicit expression in étale coordinates of $\rho$, using the expression of $R_{k}$ given in $\$ 4$.

Now, let $Z=\left\{Z_{k}\right\}$ be a pseudogroup on $X$, with $Z_{k}$ a closed subvariety of $J_{k}^{*}(X)$. Restricting $X$ if necessary, we can suppose that all the $Z_{k}$ are smooth, and the maps $Z_{k} \rightarrow Z_{\ell}(0 \leq \ell \leq k)$ are smooth and surjective. Let $L_{k}=$ Lie $Z_{k}$ be the normal bundle of the identity on $Z_{k}$. Then $L_{k}$ is a vector subbundle of $J_{k} T$, and the sections of its dual $L_{k}^{*}$ are a quotient $L_{k}^{*}$ of $D_{k} \otimes_{\mathcal{O}} \Omega^{1}$; the collection of the $L_{k}^{*}$ is a $D$-module $L^{*}$ (similarly, for each $k \geq 0$, the first prolongation $p_{1} L_{k}$ contains $L_{k+1}$ ).

Let now $\widetilde{Z}_{k}$ be the equivalence relation on $R_{k}$ corresponding to $Z_{k}$. Then the description of $L_{k}$ in terms of $\widetilde{Z}_{k}$ is the following: we take (locally on $X$ ) the vector fields on $R_{k}$ which are tangent to the equivalence classes of $\widetilde{Z}_{k}$, and are $\Gamma_{k}$-invariant (note that $\widetilde{Z}_{k}$ is stable by $\Gamma_{k}$ ). If $\widetilde{Z}_{k}$ is given by a projection $R_{k} \stackrel{\pi}{\rightarrow} S_{k}$, this means that we take the vector fields on $R_{k}$ which are $\Gamma_{k}$-invariant and tangent to the fibers of $\pi$.

To apply this to our situation we need one more definition. Let $\xi$ be the given vector field on $X$. To $\xi$ we can associate $D_{k} \xi$, i.e. $D_{k} \otimes_{\mathcal{O}} \Omega^{1} / P, P$ the sub- $\mathcal{O}_{X}$-module annihilating $\xi$. Outside of the singularities of $\xi$, the dual over $\mathcal{O}_{X}$ is the rank one bundle $J_{k} \xi$ generated by $j_{k} \xi$ over $X$. We denote the direct limit of $D_{k} \xi$ by $D \xi$, and the inverse limit of $J_{k} \xi$ by $J \xi$. Then, the theorem is the following.

THEOREM 5.1. If $G(\xi) \neq E(\xi)$, then Lie $E(\xi)=\operatorname{Lie} G(\xi) \oplus J \xi$.

It is sufficient to prove this for every $k$. Write $E(\xi)=\left\{Z_{k}\right\}, Z_{k} \subset J_{k}^{*}(X)$, and similarly $G(\xi)=\left\{Z_{k}^{\prime}\right\}$; suppose $Z_{k}^{\prime} \neq Z_{k}$. As, by definition, $Z_{k}$ is the smallest subgroupoid of $J_{k}^{*}(X)$ whose Lie algebra contains $j_{k} \xi$, we have $j_{k} \xi \in \operatorname{Lie} Z_{k}, j_{k} \xi \notin$ Lie $Z_{k}^{\prime}$.

On the other hand, the description of Lie $G(\xi)$ and Lie $E(\xi)$ in terms of first integrals, and the results of $\$ 4$ show that Lie $Z_{k}^{\prime}$ has codimension one in Lie $Z_{k}$, as vector bundles on $X$. Therefore, Lie $Z_{k}=$ Lie $Z_{k}^{\prime} \oplus J_{k} \xi$. This proves the theorem.

This result explains what we have obtained in the examples: either $\xi$ is a solution of Lie $G(\xi)$, and $G(\xi)=E(\xi)$, or the solutions of Lie $E(\xi)$ are obtained by adding $\xi$ to the solutions of Lie $G(\xi)$.

REMARK 5.2. The same result holds for $\frac{\partial}{\partial t}$ replaced by $c t \frac{\partial}{\partial t}$, with the same proof. I omit the details.

6. Generalization. I just sketch the results. They are based on the following beautiful result by Rosenlicht $\mathrm{Ro}$.

THEOREM 6.1. Let $\xi$ be a vector field on $X$ as before, and take $\varphi \in \mathbb{C}(t), \varphi \neq 0$. Denote by $\eta$ the vector field $\xi+\frac{1}{\varphi} \frac{\partial}{\partial t}$ on $X \times \mathbb{C}$. Let $K$ (resp. L) be the subfield of $\mathbb{C}(X)$ (resp. $\mathbb{C}(X \times \mathbb{C})$ of first integrals of $\xi$ (resp. $\eta)$. Then $K=L$ unless $\varphi$ has one of the following forms:

(i) $\varphi=\psi^{\prime}, \psi \in \mathbb{C}(t)$.

(ii) $\varphi=c \frac{\psi^{\prime}}{\psi}, \psi \in \mathbb{C}(t)$. 
Consider now the Galois pseudogroup of the foliation defined by $\eta$, and denote by $G(\xi, \varphi)$, its restriction to $t=a$, for a general value $a \in \mathbb{C}$. Using the arguments of prolongation of 4 and the preceding theorem, we get the following result.

Proposition 6.2. If $\varphi$ does not belong to the exceptional cases (i) or (ii), then $G(\xi, \varphi)=$ $E(\xi)$.

This is precisely the result mentioned in Example 2(iv).

It remains to analyze the exceptional cases. Suppose we are in case (i). Then we remark that the map $X \times \mathbb{C} \rightarrow X \times \mathbb{C},(x, t) \mapsto(x, s=\psi(t))$ maps $\eta$ to the vector field $\xi+\frac{\partial}{\partial s}$. From results by Casale on the behavior of the Galois pseudogroup under projections, it follows that $G(\xi, \varphi)=G(\xi, 1)$ (see [Ca1]). Therefore, we are reduced to a case already studied.

Of course, if $f \in \mathbb{C}\left(R_{k}\right)$ satisfies $\left(R_{k} \xi\right) f=1$, then $f-\psi$ is a first integral of $R_{k} \xi+\frac{1}{\varphi} \frac{\partial}{\partial t}$, or, equivalently, a differential invariant of $\eta$. But the preceding result shows that, actually, $(f-\psi)$ generates the "new" differential invariants of $\eta$.

The case $\varphi=c \frac{\psi^{\prime}}{\psi}$ is treated similarly. I omit the details.

\section{References}

[Ca1] G. Casale, Une preuve galoisienne de l'irréductibilité au sens de Nishioka-Umemura de la première équation de Painlevé, Astérisque 323 (2009), 83-100.

[Ca2] G. Casale, Morales-Ramis theorems via Malgrange pseudogroup, Ann. Inst. Fourier (Grenoble) 59 (2009), 2593-2610.

[Gr] A. Grothendieck, Techniques de construction et théorèmes d'existence en géométrie analytique, Sém. Cartan 1960-1961, Benjamin, 1967, exposés 7-17.

[Ko] E. R. Kolchin, Differential Algebra and Algebraic Groups, Pure and Applied Mathematics 54, Academic Press, New York, 1973.

[Ma1] B. Malgrange, Le groupö̈de de Galois d'un feuilletage, in: Monographies de l'Enseignement Math., Genève 38 (2001), 465-501.

[Ma2] B. Malgrange, Pseudogroupes de Lie et théorie de Galois différentielle, http://www. ihes.fr/prepublications $\mathrm{m} / 10 / 11$.

[Ol] P. J. Olver, Equivalence, Invariants, and Symmetry, Cambridge University Press, 1995.

[Ro] M. Rosenlicht, The nonminimality of the differential closure, Pacific J. Math. 52 (1974), 529-537.

[vP-Si] M. van der Put and M. F. Singer, Galois Theory of Linear Differential Equations, Grundlehren der Mathematischen Wissenschaften 328, Springer, Berlin, 2003. 